\title{
ON COMPACTNESS OF LATTICES
}

\author{
CARMEN D. VLAD
}

Received 13 July 2004 and in revised form 27 June 2005

Let $\mathbf{X}$ be an arbitrary set and $\mathbf{L}$ a lattice of subsets of $\mathbf{X}$. We denote by $\mathbf{I}(\mathbf{L})$ the set of those zero-one-valued nontrivial, finitely additive measures on $\mathbf{A}(\mathbf{L})$, the algebra generated by $\mathbf{L}$, and we introduce other subsets of $\mathbf{I}(\mathbf{L})$. We study compactness/normality properties either relating to a single lattice $\mathbf{L}$ or relating to a pair of lattices $\mathbf{L}_{1} \subset \mathbf{L}_{2}$ of subsets of $\mathbf{X}$.

\section{Introduction}

In this paper, we present a study of various concepts pertaining to compactness properties of a lattice. Special cases include such notions as countably compactness, almost countably compactness, and countably paracompactness. Some investigations in these matters have been done in $[2,7,14]$. We go beyond these results and introduce new subsets of $\mathbf{I}(\mathbf{L})$ and their lattices to investigate. This way, we investigate the topological-like properties of lattices through measurability techniques.

We consider $\mathbf{X}$ to be an arbitrary nonempty set and $\mathbf{L}$ a lattice of subsets of $\mathbf{X}$ such that $\varnothing, \mathbf{X} \in \mathbf{L}$. By $\mathbf{A}(\mathbf{L})$ we denote the algebra of subsets of $\mathbf{X}$ generated by $\mathbf{L}$, and $\mathbf{I}(\mathbf{L})$ denotes those nontrivial, zero-one-valued, finitely additive measures on $\mathbf{A}(\mathbf{L})$.

We begin with some standard notations and terminology that will be used throughout the paper. Our notations and terminology are consistent with those in the literature (see, e.g., $[1,2,8,14])$ and are added mainly for the reader's convenience.

We then proceed to analyze interrelations between these various concepts as indicated above. In the last part of the paper we wish to extend some lattice compactness results to the important situation of two lattices $\mathbf{L}_{1} \subset \mathbf{L}_{2}$ of subsets of $\mathbf{X}$ and investigate separation properties in connection to various aspects of compactness properties.

Throughout the paper we treat almost all cases by general measure-theoretic techniques, the advantage being that much of the results obtained for the special case of zeroone valued measures can easily be extended to the case of arbitrary measures on $\mathbf{A}(\mathbf{L})$ which are nonnegative, finite, finitely additive, not necessarily two-valued measures of $\mathbf{I}(\mathbf{L})$ (as in the case of Theorem 5.4). 


\section{Lattice terminology}

In this section, we introduce the notation and terminology that will be used throughout the paper. All is fairly standard and we include it for the reader's convenience.

Let $\mathbf{X}$ be an arbitrary nonempty set and $\mathbf{L}$ a lattice of subsets of $\mathbf{X}$ such that $\varnothing, \mathbf{X} \in \mathbf{L}$. A lattice $\mathbf{L}$ is a partially ordered set any two elements $(x, y)$ of which have both $\sup (x, y)$ and $\inf (x, y)$.

$\mathbf{A}(\mathbf{L})$ is the algebra generated by $\mathbf{L} ; \delta(\mathbf{L})$ is the lattice of all countable intersections of sets from $\mathbf{L} ; \sigma(\mathbf{L})$ is the $\sigma$-algebra generated by $\mathbf{L} ; s(\mathbf{L})$ is the family of Souslin sets obtained from the lattice $\mathbf{L}$ by the lattice Souslin operations.

Definition 2.1. Some lattice definitions in a point-set framework, analogous with the general topology definitions, which will be used to obtain lattice-topological conditions/ proofs of the measure-theoretic results, are introduced here.

The lattice $\mathbf{L}$ is called

(i) $\boldsymbol{\delta}$-lattice if $\mathbf{L}$ is closed under countable intersections;

(ii) disjunctive if for $x \in \mathbf{X}$ and $A \in \mathbf{L}$ such that $x \notin A$ there exists $B \in \mathbf{L}$ with $x \in B$ and $A \cap B=\varnothing$;

(iii) separating (or $\mathbf{T}_{1}$ ) if $x, y \in \mathbf{X}$ and $x \neq y$ implies that there exists $A \in \mathbf{L}$ such that $x \in A, y \notin A$

(iv) (topologically) normal if and only if for any disjoint sets $A, B \in \mathbf{L}$, there exist $C, D \in$ L with $A \subset C^{\prime}, B \subset D^{\prime}$ and $C^{\prime} \cap D^{\prime}=\varnothing$ (where prime denotes the complement);

(v) (topologically) compact if for any collection $\left\{L_{\alpha}\right\}$ of sets of $\mathbf{L}$ with $\cap L_{\alpha}=\varnothing$, there exists a finite subcollection with empty intersection;

(vi) (topologically) countably compact if for any countable collection $\left\{L_{\alpha}\right\}$ of sets of $\mathbf{L}$ with $\cap L_{\alpha}=\varnothing$, there exists a finite subcollection with empty intersection;

(vii) countably paracompact if and only if for any sequence $\left\{A_{n}\right\}$ of sets of $\mathbf{L}$ with $A_{n} \downarrow$ $\varnothing$, there exists a sequence $\left\{B_{n}\right\}$ of sets of $\mathbf{L}$ with $A_{n} \subset B_{n}^{\prime}$ for all $n$ and $B_{n}^{\prime} \downarrow \varnothing$,

(viii) complement generated if and only if $L \in \mathbf{L}$ implies $L=\cap L_{n}^{\prime}, L_{n} \in \mathbf{L}$.

\section{Measure terminology}

The thrust of the paper is towards measure-theoretic approach, therefore we introduce now some necessary definitions and notations. Regarding the general topological concepts and preliminaries relating to measure, the reader is referred to the works of Alexandroff [1], Halmos [9], and Munroe [12]. Regarding the results which are mentioned in this work without explanations, they can be found in the cited previous papers of the author or of the other researchers.

Definition 3.1. (i) $\mathbf{I}(\mathbf{L})$ is the set of nontrivial zero-one-valued, finitely additive measures on $\mathbf{A}(\mathbf{L})$.

(ii) $\mathbf{I}_{\mathbf{R}}(\mathbf{L})$ is the set of $\mathbf{L}$-regular measures of $\mathbf{I}(\mathbf{L})$, where $\mu \in \mathbf{I}(\mathbf{L})$ is $\mathbf{L}$-regular if for any $A \in \mathbf{A}(\mathbf{L}), \mu(A)=\sup \{\mu(L) / L \subset A, L \in \mathbf{L}\}$.

(iii) $\mathbf{I}_{\sigma}(\mathbf{L})$ is the set of $\sigma$-smooth measures on $\mathbf{L}$ of $\mathbf{I}(\mathbf{L})$, where $\mu \in \mathbf{I}(\mathbf{L})$ is $\sigma$-smooth on

$\mathbf{L}$ (countably additive) if for all sequences $\left\{L_{n}\right\}$ of sets of $\mathbf{L}$ with $L_{n} \downarrow \varnothing, \mu\left(L_{n}\right) \rightarrow 0$.

(iv) $\mathbf{I}^{\sigma}(\mathbf{L})$ is the set of $\sigma$-smooth measures on $\mathbf{A}(\mathbf{L})$ of $\mathbf{I}(\mathbf{L})$.

(v) $\mathbf{I}^{\sigma}{ }_{\mathbf{R}}(\mathbf{L})$ is the set of $\mathbf{L}$-regular measures of $\mathbf{I}^{\sigma}(\mathbf{L})$. 
Note that

(i) for any $\mu \in \mathbf{I}(\mathbf{L})$, there exists $\nu \in \mathbf{I}_{\mathbf{R}}(\mathbf{L})$ such that $\mu \leq \nu$ on $\mathbf{L}$;

(ii) for any $\mu \in \mathbf{I}(\mathbf{L})$, there exists $\lambda \in \mathbf{I}_{\mathbf{R}}\left(\mathbf{L}^{\prime}\right)$ such that $\mu \leq \lambda$ on $\mathbf{L}^{\prime}$. (For details, see [10].)

For the general case, we will assume with no loss of generality that all measures are nonnegative. So, $\mathbf{M}(\mathbf{L})$ denotes those nonnegative, finite, finitely additive measures on $\mathbf{A}(\mathbf{L})$.

As in the zero-one-valued case, we also have $\mathbf{M}_{\mathbf{R}}(\mathbf{L}), \mathbf{M}_{\sigma}(\mathbf{L}), \mathbf{M}^{\sigma}(\mathbf{L}), \mathbf{M}^{\sigma}{ }_{\mathbf{R}}(\mathbf{L})$.

We note here that the condition that the measures in $\mathbf{M}(\mathbf{L})$ are nonnegative is not restrictive and we do not loose the generality of our statements, since a measure on $\mathbf{A}(\mathbf{L})$ is the difference of nonnegative, finite, finitely additive measures on $\mathbf{A}(\mathbf{L})$, that is, elements of $\mathbf{M}(\mathbf{L})$.

\section{The support of a measure}

The notion of compactness of lattices includes special cases as countably compactness, almost countably compactness, and countably paracompactness and we develop measuretheoretic results to show systematically how to obtain compactness/normality relations. Since the measure arguments are simpler and more natural for such problems, we make use of the support of a zero-one-valued measure, the intersection of all sets of $\mathbf{L}$ of measure one. Moreover, various lattice topological properties already introduced in Definition 2.1 can be characterized in a measure theoretic way as follows.

Definition 4.1. The support of $\mu \in \mathbf{I}(\mathbf{L})$ is $\mathbf{S}(\mu)=\cap\{L \in \mathbf{L} / \mu(L)=1\}$.

Note that in the general case of a measure $\mu \in \mathbf{M}(\mathbf{L})$, the support is defined by

$$
\mathbf{S}(\mu)=\cap\{L \in \mathbf{L} / \mu(L)=\mu(\mathbf{X})\}
$$

In terms of measures and support, now rephrase some of the previous definitions and introduce new definitions. These results are known, they appear in $[3,9,11]$. The lattice $\mathbf{L}$ is called

(i) (measurably) normal if and only if for each $\mu \in \mathbf{I}(\mathbf{L})$, there exists a unique $\nu \in$ $\mathbf{I}_{\mathbf{R}}(\mathbf{L})$ such that $\mu \leq \nu(\mathbf{L})$, that is, $\mu(L) \leq \nu(L)$ for all $L \in \mathbf{L}$;

(ii) strongly normal if for $\mu, \mu_{1}, \mu_{2} \in \mathbf{I}(\mathbf{L})$ with $\mu \leq \mu_{1}, \mu_{2}$ on $\mathbf{L}$, the following inequalities are true:

$$
\mu_{1} \leq \mu_{2} \quad \text { on } \mathbf{L} \quad \text { or } \quad \mu_{2} \leq \mu_{1} \quad \text { on } \mathbf{L} \text {; }
$$

(iii) (measurably) compact if and only if $S(\mu) \neq \varnothing$ for every $\mu \in \mathbf{I}_{\mathbf{R}}(\mathbf{L})$;

(iv) countably compact if and only if $\mathbf{I}_{\mathbf{R}}(\mathbf{L})=\mathbf{I}^{\sigma}{ }_{\mathbf{R}}(\mathbf{L})$. It should be pointed out that in the relevant literature, this definition is sometimes replaced by the equivalent one $\mathbf{I}(\mathbf{L})=\mathbf{I}_{\sigma}(\mathbf{L}) ;$

(v) almost countably compact if $\mu \in \mathbf{I}_{\mathbf{R}}\left(\mathbf{L}^{\prime}\right)$ implies $\mu \in \mathbf{I}_{\sigma}(\mathbf{L})$. 


\section{Compact lattices}

We begin with some preliminary, easily proven results on interrelations of countably compactness, almost countably compactness, countably paracompactness, and normality. The approach is measure theoretic and is done either in the general case or in the case of zero-one-valued measures. The obtained results generalize, strengthen, and complement results of $[3,10,14]$. General properties and representations theorems for countably compact lattices were studied extensively in [5] and one may want to consult this paper for more information.

THeOREM 5.1. If $\mathbf{L}$ is countably compact then it is almost countably compact.

Proof. Let $\mu \in \mathbf{I}_{\mathbf{R}}\left(\mathbf{L}^{\prime}\right)$; then $\mu \in \mathbf{I}(\mathbf{L})$, and since $\mathbf{L}$ is countably compact, $\mathbf{I}(\mathbf{L})=\mathbf{I}_{\sigma}(\mathbf{L})$.

Hence $\mu \in \mathbf{I}_{\sigma}(\mathbf{L})$.

We note without proof the following well-known results (see [3]).

Theorem 5.2. (a) If $\mathbf{L}$ is countably paracompact then $\mathbf{I}_{\sigma}\left(\mathbf{L}^{\prime}\right) \subset \mathbf{I}_{\sigma}(\mathbf{L})$.

(b) If $\mathbf{L}$ is countably paracompact and normal and if $\mu, \nu \in \mathbf{I}(\mathbf{L})$ and $\mu \leq \nu(\mathbf{L})$ then $\mu \in \mathbf{I}_{\sigma}(\mathbf{L}) \Rightarrow \nu \in \mathbf{I}_{\sigma}(\mathbf{L})$.

(c) If $\mathbf{L}$ is countably paracompact and normal and if for any $\mu \in \mathbf{I}^{\sigma}{ }_{\mathbf{R}}(\mathbf{L}), \mathbf{S}(\mu) \neq \varnothing$ then for any $\mu \in \mathbf{I}_{\sigma}(\mathbf{L}), \mathbf{S}(\mu) \neq \varnothing$.

THeOREM 5.3. If $\mathbf{L}$ is normal, countably paracompact, and almost countably compact, then $\mathbf{L}$ is countably compact.

Proof. Let $\mu \in \mathbf{I}_{\mathbf{R}}(\mathbf{L})$ be an arbitrary L-regular measure, so $\mu \in \mathbf{I}\left(\mathbf{L}^{\prime}\right)$. Then $\mu \leq \nu\left(\mathbf{L}^{\prime}\right)$, where $v \in \mathbf{I}_{\mathbf{R}}\left(\mathbf{L}^{\prime}\right)$ (see the note of Definition 3.1). Since $\mathbf{L}$ is almost countably compact, $\nu \in \mathbf{I}_{\sigma}(\mathbf{L})$.

But $\nu \leq \mu(\mathbf{L})$ and since $\mathbf{L}$ is normal and countably paracompact, it follows that $\mu \in$ $\mathbf{I}^{\sigma}{ }_{\mathbf{R}}(\mathbf{L})$, therefore $\mathbf{I}_{\mathbf{R}}(\mathbf{L})=\mathbf{I}^{\sigma}{ }_{\mathbf{R}}(\mathbf{L})$.

The next theorem is interesting because it extends the almost countably compact property defined in case of zero-one-valued measures on $\mathbf{A}(\mathbf{L})$ to the general case of measures on $\mathbf{A}(\mathbf{L})$. In other words, if $\mathbf{L}$ is almost countably compact with respect to zero-onevalued-measures, then it is almost countably compact with respect to any measure.

Theorem 5.4. Let $\mathbf{L}$ be almost countably compact and let $\mu \in \mathbf{M}_{\mathbf{R}}\left(\mathbf{L}^{\prime}\right)$. Then $\mu \in \mathbf{M}_{\sigma}(\mathbf{L})$.

Proof. Suppose $\mu \notin \mathbf{M}_{\sigma}(\mathbf{L})$. Then there is a sequence $A_{n} \downarrow \varnothing, A_{n} \in \mathbf{L}$, but $\mu\left(A_{n}\right)$ not convergent to 0 . Since $\mu \in \mathbf{M}_{\mathbf{R}}\left(\mathbf{L}^{\prime}\right)$, there exist $B_{n} \in \mathbf{L}, A_{n} \supset B_{n}^{\prime}$, and $\mu\left(A_{n}\right) \cong \mu\left(B_{n}^{\prime}\right)$ not convergent to 0 . we may assume $B_{n}^{\prime} \neq \varnothing$ for any $\mathrm{n}$ and $B_{n}^{\prime} \downarrow \varnothing$.

Clearly, $\left\{B_{n}^{\prime}\right\}$ has the finite intersection property, that is, $\cap\left\{B_{n}^{\prime} \in \mathbf{L} / \mu\left(B_{n}^{\prime}\right)=1\right\} \neq \varnothing$.

Therefore there exists a zero-one $\mathbf{L}^{\prime}$-regular measure, call it $\lambda, \lambda \in \mathbf{I}_{\mathbf{R}}\left(\mathbf{L}^{\prime}\right)$, such that $\lambda\left(B_{n}^{\prime}\right)=1$ for all $n$. Since $A_{n} \supset B_{n}^{\prime}$ it follows that $\lambda\left(A_{n}\right)=1$ for all $n$.

But $A_{n} \downarrow \varnothing$; a contradiction since $\mathbf{L}$ is almost countably compact and then $\lambda$ should be countably additive on $\mathbf{L}$.

Theorem 5.5. Let $\mathbf{L}$ be a normal lattice and let $\mu \in \mathbf{M}(\mathbf{L}), v \in \mathbf{M}_{\mathbf{R}}(\mathbf{L})$ with $\mu \leq v$ on $\mathbf{L}$ and $\mu(\mathbf{X})=\nu(\mathbf{X})$. (This is just a generalization of the remark made in the note of Definition 3.1.) Then $\nu\left(L^{\prime}\right)=\sup \left\{\mu(\widetilde{L}), \widetilde{L} \subset L^{\prime}, L, \widetilde{L} \in \mathbf{L}\right\}$. 
Proof. Let $L \in \mathbf{L}$ and $\varepsilon>0$. Since $v \in \mathbf{M}_{\mathbf{R}}(\mathbf{L})$, there exists $A \subset L^{\prime}, A \in \mathbf{L}$ such that $\nu\left(L^{\prime}\right)-$ $\varepsilon<\nu(A)$. Since $\mathbf{L}$ is (topologically) normal, there exist $B, C \in \mathbf{L}$ such that $A \subset B^{\prime} \subset C \subset$ $L^{\prime}$, hence $\nu(A) \leq \nu\left(B^{\prime}\right) \leq \mu\left(B^{\prime}\right) \leq \mu(C) \leq \nu(C) \leq \nu\left(L^{\prime}\right)$ and then $\nu\left(L^{\prime}\right)-\mu(C) \leq \nu\left(L^{\prime}\right)-$ $\nu(A)<\varepsilon$, that is, $\nu\left(L^{\prime}\right)<\mu(C)+\varepsilon$, $\varepsilon$ arbitrary small.

Therefore $\nu\left(L^{\prime}\right)=\sup \left\{\mu(\widetilde{L}), \widetilde{L} \subset L^{\prime}, L, \widetilde{L} \in \mathbf{L}\right\}$.

Based on the above result, we prove now the next theorem which shows that the normality of $\mathbf{L}$ implies that the usual dominating $\mathbf{L}$-regular measure is also $\sigma$-smooth on $\mathbf{L}^{\prime}$, the lattice of the complements of the sets of $\mathbf{L}$.

Theorem 5.6. Let $\mathbf{L}$ be a normal lattice and let $\mu \in \mathbf{M}_{\sigma}(\mathbf{L}), v \in \mathbf{M}_{\mathbf{R}}(\mathbf{L})$ with $\mu \leq \nu$ on $\mathbf{L}$ and $\mu(\mathbf{X})=v(\mathbf{X})$. Then $v \in \mathbf{M}_{\sigma}\left(\mathbf{L}^{\prime}\right)$.

Proof. Let $L_{n}^{\prime} \downarrow \varnothing, L_{n} \in \mathbf{L}$. Since $\nu \in \mathbf{M}_{\mathbf{R}}(\mathbf{L})$ and $\mu \in \mathbf{M}_{\sigma}(\mathbf{L})$ with $\mu \leq \nu$ on $\mathbf{L}$, we have by Theorem $5.5 v\left(L^{\prime}\right)=\sup \left\{\mu(\tilde{L}), \tilde{L} \subset L^{\prime}, L, \tilde{L} \in \mathbf{L}\right\}$. Therefore, given $\varepsilon>0$, there exist $\tilde{L}_{n} \subset$ $L_{n}^{\prime}, \widetilde{L}_{n} \in \mathbf{L}$ such that $\nu\left(L_{n}^{\prime}\right)<\mu\left(\tilde{L}_{n}\right)+\varepsilon / 2$. We may assume $\widetilde{L}_{n} \downarrow$, and since $\widetilde{L}_{n} \subset L_{n}^{\prime} \downarrow \varnothing$, it follows, $\tilde{L}_{n} \downarrow \varnothing$. Since $\mu \in \mathbf{M}_{\sigma}(\mathbf{L}), \mu\left(\tilde{L}_{n}\right)<\varepsilon / 2$ for all $n \geq N(\varepsilon)$, hence $\nu\left(L_{n}^{\prime}\right)<\varepsilon$ for all $n \geq N(\varepsilon)$, that is, $\nu \in \mathbf{M}_{\sigma}\left(\mathbf{L}^{\prime}\right)$.

Next, we investigate the case of the lattice $\mathbf{L}$ being normal and countably compact and show that any $\mathbf{L}$-regular measure is $\mathbf{L}^{\prime}$-countably additive.

Theorem 5.7. Let $\mathbf{L}$ be normal and almost countably compact. Then $\mathbf{M}_{\mathbf{R}}(\mathbf{L}) \subset \mathbf{M}_{\sigma}\left(\mathbf{L}^{\prime}\right)$.

Proof. Let $\mu \in \mathbf{M}_{\mathbf{R}}(\mathbf{L})$. Then, according to the note following Definition 3.1 applied to the general case of arbitrary measures, we have $\mu \leq \rho \in \mathbf{M}_{\mathbf{R}}\left(\mathbf{L}^{\prime}\right)$ on $\mathbf{L}^{\prime}$ and $\rho(\mathbf{X})=\mu(\mathbf{X})$. Hence $\rho \leq \mu$ on $\mathbf{L}$ and $\rho \in \mathbf{M}_{\sigma}(\mathbf{L})$ since $\mathbf{L}$ is almost countably compact. But $\rho \in \mathbf{M}_{\sigma}(\mathbf{L})$ and $\mu \in \mathbf{M}_{\mathbf{R}}(\mathbf{L})$ and $\rho \leq \mu$ on $\mathbf{L}$, therefore, $\mathbf{L}$ being normal, it follows by the previous theorems that $\mu \in \mathbf{M}_{\sigma}\left(\mathbf{L}^{\prime}\right)$.

The next theorem strengthens the result of Theorem 5.6 in the special case of zeroone-valued measures. We give here a slightly different proof.

Theorem 5.8. Let $\mathbf{L}$ be a normal lattice and let $\mu \in \mathbf{I}_{\sigma}(\mathbf{L})$ and $\nu \in \mathbf{I}_{\mathbf{R}}(\mathbf{L})$ such that $\mu \leq \nu(\mathbf{L})$. Then $v \in \mathbf{I}_{\sigma}\left(\mathbf{L}^{\prime}\right)$. If $\mathbf{L}$ is also countably paracompact then $\nu \in \mathbf{I}^{\sigma}{ }_{\mathbf{R}}(\mathbf{L})$.

Proof. Let $L_{n}^{\prime} \downarrow \varnothing, L_{n} \in \mathbf{L}$ and suppose $\nu\left(L_{n}^{\prime}\right)=1$ for all $n$. Since $v \in \mathbf{I}_{\mathbf{R}}(\mathbf{L})$, there exist $\widetilde{L}_{n} \subset L_{n}^{\prime}, \widetilde{L}_{n} \in \mathbf{L}, v\left(\tilde{L}_{n}\right)=1$ for all $n$ and $\tilde{L}_{n} \downarrow$.

$\mathbf{L}$ is normal, therefore there exist $A_{n}^{\prime}, B_{n}^{\prime}$ such that

$$
L_{n} \subset A_{n}^{\prime}, \quad \tilde{L}_{n} \subset B_{n}^{\prime}, \quad A_{n}^{\prime} \cap B_{n}^{\prime}=\varnothing, \quad A_{n}, B_{n} \in \mathbf{L} .
$$

Consider the sequence of inclusions

$$
\tilde{L}_{n} \subset B_{n}^{\prime} \subset A_{n} \subset L_{n}^{\prime}
$$

Since $L_{n}^{\prime} \downarrow \varnothing$, we may assume with no loss of generality that

$$
\tilde{L}_{n}, B_{n}^{\prime}, A_{n} \downarrow \varnothing .
$$

Now, $\mu \in \mathbf{I}_{\sigma}(\mathbf{L})$ implies $\mu\left(B_{n}^{\prime}\right) \rightarrow 0$. 
But $\mu\left(B_{n}^{\prime}\right) \geq \nu\left(B_{n}^{\prime}\right)=1$, a contradiction.

Next let $\mathbf{L}$ be also countably paracompact and let

$$
\nu \in \mathbf{I}_{\sigma}\left(\mathbf{L}^{\prime}\right), \quad B_{n} \in \mathbf{L}, \quad B_{n} \downarrow \varnothing .
$$

There exist $A_{n} \in \mathbf{L}, B_{n} \subset A_{n}^{\prime} \downarrow \varnothing$, and $\nu\left(B_{n}\right) \leq \nu\left(A_{n}^{\prime}\right) \rightarrow 0$.

Therefore $\nu \in \mathbf{I}^{\sigma}{ }_{\mathbf{R}}(\mathbf{L})$.

\section{Application: semiseparation of lattices}

In this section, we wish to extend some lattice compactness results to the case of $\mathbf{L}_{1} \subset \mathbf{L}_{2}$ lattices of subsets of $\mathbf{X}$. In particular we consider the case of either lattice being countably compact and the case of $\mathbf{L}_{2}$ being $\mathbf{L}_{1}$-countably bounded or $\mathbf{L}_{1}$-countably paracompact. Finally, we introduce a domination condition $(\sigma)$ on smoothness of zero-onevalued measures and use it to obtain new results on the interrelations between normality, semiseparation, and compactness of lattices.

Definition 6.1. Next consider that $\mathbf{X}$ is an abstract set and that $\mathbf{L}_{1} \subset \mathbf{L}_{2}$ are lattices of subsets of $\mathbf{X}$. We say that

(i) $\mathbf{L}_{1}$ semiseparates $\mathbf{L}_{2}$ if for $A \in \mathbf{L}_{1}, B \in \mathbf{L}_{2}$ with $A \cap B=\varnothing$, there exists $C \in \mathbf{L}_{1}$ such that $B \subset C$ and $A \cap C=\varnothing$;

(ii) $\mathbf{L}_{1}$ separates $\mathbf{L}_{2}$ if for $A, B \in \mathbf{L}_{2}$ with $A \cap B=\varnothing$, there exists $C, D \in \mathbf{L}_{1}$ such that $A \subset C, B \subset D$, and $C \cap D=\varnothing$;

(iii) $\mathbf{L}_{2}$ is $\mathbf{L}_{1}$-countably paracompact if for $A_{n} \in \mathbf{L}_{2}, n=1,2, \ldots$, with $A_{n} \downarrow \varnothing$, there exists $B_{n} \in \mathbf{L}_{1}, n=1,2, \ldots$, such that $A_{n} \subset B_{n}^{\prime}$ and $B_{n}^{\prime} \downarrow \varnothing$;

(iv) $\mathbf{L}_{2}$ is $\mathbf{L}_{1}$-countably bounded if for $A_{n} \in \mathbf{L}_{2}, n=1,2, \ldots$, with $A_{n} \downarrow \varnothing$, there exists $B_{n} \in \mathbf{L}_{1}, n=1,2, \ldots$, such that $A_{n} \subset B_{n}$ and $B_{n} \downarrow \varnothing$.

TheOrem 6.2. Let $\mathbf{L}_{1} \subset \mathbf{L}_{2}$ be lattices of subsets of $\mathbf{X}$. If $\mathbf{L}_{2}$ is almost countably compact then $\mathbf{L}_{1}$ is almost countably compact.

Proof. Let $\mu \in \mathbf{I}_{\mathbf{R}}\left(\mathbf{L}_{1}^{\prime}\right)$ and extend it to $\nu \in \mathbf{I}_{\mathbf{R}}\left(\mathbf{L}_{2}^{\prime}\right)$. $\mathbf{L}_{2}$ almost countably compact implies $\mathbf{I}_{\mathbf{R}}\left(\mathbf{L}_{2}^{\prime}\right)=\mathbf{I}_{\sigma}\left(\mathbf{L}_{2}\right)$, hence $\nu \in \mathbf{I}_{\sigma}\left(\mathbf{L}_{2}\right)$. Clearly, $\nu \in \mathbf{I}_{\sigma}\left(\mathbf{L}_{1}\right)$, that is, $\mu \in \mathbf{I}_{\sigma}\left(\mathbf{L}_{1}\right)$, therefore $\mathbf{L}_{1}$ is almost countably compact.

Theorem 6.3. Let $\mathbf{L}_{1} \subset \mathbf{L}_{2}$ be lattices of subsets of $\mathbf{X}$ and suppose that $\mathbf{L}_{1}^{\prime}$ semiseparates $\mathbf{L}_{2}^{\prime}$. Suppose that $\mathbf{L}_{1}$ is almost countably compact and that if $B_{n}$ is a sequence of sets of $\mathbf{L}_{2}$ with $B_{n} \downarrow \varnothing$ then there exists the sequence of sets $A_{n} \in \mathbf{L}_{1}$ with $B_{n} \supset A_{n} \downarrow \varnothing$ and $\lambda\left(B_{n}\right)=\lambda\left(A_{n}\right)$ where $\lambda \in \mathbf{I}_{\mathbf{R}}\left(\mathbf{L}_{2}^{\prime}\right)$. Then $\mathbf{L}_{2}$ is almost countably compact.

Proof. Let $\lambda \in \mathbf{I}_{\mathbf{R}}\left(\mathbf{L}_{2}^{\prime}\right)$ and consider the restriction $\lambda \mid L_{1}^{\prime}=\mu$. By semiseparation, $\mu \in$ $\mathbf{I}_{\mathbf{R}}\left(\mathbf{L}_{1}^{\prime}\right)$ (for all the details see [6, Lemma 3.1, page 300]).

Since $\mathbf{L}_{1}$ is almost countably compact, it follows that $\mu \in \mathbf{I}_{\sigma}\left(\mathbf{L}_{1}\right)$.

Now $\lambda\left(B_{n}\right)=\lambda\left(A_{n}\right)=\mu\left(A_{n}\right)$. But $A_{n} \downarrow \varnothing$ and $\mu \in \mathbf{I}_{\sigma}\left(\mathbf{L}_{1}\right)$ implies that $\mu\left(A_{n}\right) \rightarrow 0$. Therefore $\lambda\left(B_{n}\right) \rightarrow 0$ where $B_{n} \in \mathbf{L}_{2}, B_{n} \downarrow \varnothing$. Then $\lambda \in \mathbf{I}_{\sigma}\left(\mathbf{L}_{2}\right)$ and $\mathbf{L}_{2}$ is almost countably compact. 
Theorem 6.4. Let $\mathbf{L}$ be a countably compact lattice of subsets of $\mathbf{X}$. Then $\delta(\mathbf{L})$ is countably compact and $\mathbf{L}$ separates $\delta(\mathbf{L})$.

Proof. Let $\nu \in \mathbf{I}(\delta(\mathbf{L}))$ and consider the restriction $\left.\nu\right|_{L}=\mu \in \mathbf{I}(\mathbf{L})=\mathbf{I}_{\sigma}(\mathbf{L})$, since $\mathbf{L}$ is countably compact. Clearly, $v \in \mathbf{I}_{\sigma}(\delta(\mathbf{L}))$, which proves that $\delta(\mathbf{L})$ is countably compact.

Next, to show that $\mathbf{L}$ separates $\delta(\mathbf{L})=\left\{\bigcap_{n=1}^{\infty} A_{n} / A_{n} \in L\right\}$, let

$$
\left(\bigcap_{i=1}^{\infty} A_{i}\right) \cap\left(\bigcap_{j=1}^{\infty} B_{j}\right)=\varnothing, \quad A_{i}, B_{j} \in \mathbf{L} .
$$

This implies that

$$
\left(\bigcup_{i=1}^{\infty} A_{i}^{\prime}\right) \cup\left(\bigcup_{j=1}^{\infty} B_{j}^{\prime}\right)=\mathbf{X}
$$

Then also

$$
\left(\bigcup_{i=1}^{n} A_{i}^{\prime}\right) \cup\left(\bigcup_{j=1}^{m} B_{j}^{\prime}\right)=\mathbf{X}
$$

which implies

$$
\left(\bigcap_{i=1}^{n} A_{i}\right) \cap\left(\bigcap_{j=1}^{m} B_{j}\right)=\varnothing
$$

But

$$
\bigcap_{i=1}^{\infty} A_{i} \subset \bigcap_{i=1}^{n} A_{i} \in \mathbf{L}, \quad \bigcap_{j=1}^{\infty} B_{j} \subset \bigcap_{j=1}^{m} B_{j} \in \mathbf{L},
$$

which proves separation.

Theorem 6.5. Let $\mathbf{L}_{1} \subset \mathbf{L}_{2}$ be two lattices of subsets of $\mathbf{X}$. Suppose that $\mathbf{L}_{2}$ is $\mathbf{L}_{1}$-countably bounded and that $\mathbf{L}_{1}$ is countably compact. Then $\mathbf{L}_{2}$ is countably compact.

Proof. Let $\nu \in \mathbf{I}\left(\mathbf{L}_{2}\right)$ and consider the restriction $\left.\nu\right|_{A\left(L_{1}\right)}=\mu \in \mathbf{I}\left(\mathbf{L}_{1}\right)$. If we let $B_{n} \in \mathbf{L}_{2}$ such that $B_{n} \downarrow \varnothing$, by $\mathbf{L}_{2}$ being $\mathbf{L}_{1}$-countably bounded, it follows that there exists $A_{n} \in \mathbf{L}_{1}$, $B_{n} \subset A_{n}$ for all $n$ and $A_{n} \downarrow \varnothing$. We then have $\nu\left(B_{n}\right) \leq \nu\left(A_{n}\right)=\mu\left(A_{n}\right)$. But $\mathbf{L}_{1}$ is countably compact, therefore $\mathbf{I}\left(\mathbf{L}_{1}\right)=\mathbf{I}_{\sigma}\left(\mathbf{L}_{1}\right)$, which implies $\mu\left(A_{n}\right) \rightarrow 0$.

Therefore $\mathbf{I}\left(\mathbf{L}_{2}\right)=\mathbf{I}_{\sigma}\left(\mathbf{L}_{2}\right)$, that is, $\mathbf{L}_{2}$ is countably compact.

Theorem 6.6. Let $\mathbf{L}_{1} \subset \mathbf{L}_{2}$ be two lattices of subsets of $\mathbf{X}$. Suppose that $\mathbf{L}_{2}$ is countably paracompact and that $\mathbf{L}_{1}$ separates $\mathbf{L}_{2}$. Then $\mathbf{L}_{2}$ is $\mathbf{L}_{1}$-countably paracompact and $\mathbf{L}_{1}$-countably bounded.

Proof. Because $\mathbf{L}_{2}$ is countably paracompact, given $A_{n} \in \mathbf{L}_{2}, A_{n} \downarrow \varnothing$, there exists $B_{n} \in \mathbf{L}_{2}$ such that $A_{n} \subset B_{n}^{\prime}$ (or $A_{n} \cap B_{n}=\varnothing$ ) and $B_{n}^{\prime} \downarrow \varnothing$. Since $\mathbf{L}_{1}$ separates $\mathbf{L}_{2}$, there exist $C_{n}$ and 
$D_{n} \in \mathrm{L}_{1}$, such that $A_{n} \subset C_{n}, B_{n} \subset D_{n}$ and $C_{n} \cap D_{n}=\varnothing$. But then we have the sequence of inclusions $A_{n} \subset C_{n} \subset D_{n}^{\prime} \subset B_{n}^{\prime} \downarrow \varnothing$. Therefore $A_{n} \subset D_{n}^{\prime} \downarrow \varnothing$, with $A_{n} \in \mathbf{L}_{2}$ and $D_{n} \in \mathbf{L}_{1}$, which proves that $\mathbf{L}_{2}$ is $\mathbf{L}_{1}$-countably paracompact.

On other hand, $A_{n} \subset C_{n} \downarrow \varnothing$ with $A_{n} \in \mathbf{L}_{2}$ and $C_{n} \in \mathbf{L}_{1}$, which proves that $\mathbf{L}_{2}$ is $\mathbf{L}_{1}$ countably bounded.

For the next theorems, we say that the lattice $\mathbf{L}$ satisfies the domination condition $(\sigma)$ on smoothness if for any $\mu \in \mathbf{I}_{\sigma}(\mathbf{L})$ there exists $\nu \in \mathbf{I}^{\sigma}{ }_{\mathbf{R}}(\mathbf{L})$ such that $\mu \leq \nu$ on $\mathbf{L}$.

Theorem 6.7. Let $\mathbf{L}_{1} \subset \mathbf{L}_{2}$ be two lattices of subsets of $\mathbf{X}$. Suppose that $\mathbf{L}_{1}$ satisfies condition $(\sigma)$. Then the following are true.

(a) If $\mathbf{L}_{1}$ is strongly normal and if $\mathbf{L}_{2}$ is $\mathbf{L}$-countably bounded, then $\mathbf{L}_{2}$ satisfies condition $(\sigma)$.

(b) If $\mathbf{L}_{1}$ is normal and semiseparates $\mathbf{L}_{2}$ and if $\mathbf{L}_{2}$ is $\mathbf{L}_{1}$-countably bounded, then $\mathbf{L}_{2}$ satisfies condition $(\sigma)$.

(c) If $\mathbf{L}_{1}$ is normal and semiseparates $\mathbf{L}_{2}$ and if $\mathbf{L}_{2}$ is $\mathbf{L}_{1}$-countably paracompact, then $\mathbf{L}_{2}$ satisfies condition $(\sigma)$.

Proof. Let $\nu \in \mathbf{I}_{\sigma}\left(\mathbf{L}_{2}\right)$. Then the restriction of $\nu$ to $\mathbf{A}\left(\mathbf{L}_{1}\right)$, call it $\mu$, is clearly countably additive and we have $\left.\nu\right|_{A\left(L_{1}\right)}=\mu \in \mathbf{I}_{\sigma}\left(\mathbf{L}_{1}\right)$, and since $\mathbf{L}_{1}$ satisfies condition $(\sigma)$, there exists $\lambda \in \mathbf{I}^{\sigma}{ }_{\mathbf{R}}\left(\mathbf{L}_{1}\right)$ such that $\mu \leq \lambda$ on $\mathbf{L}_{1}$. Let $\rho \in \mathbf{I}_{\mathbf{R}}\left(\mathbf{L}_{2}\right)$. Then, according to the note following Definition 3.1, the measures $\nu$ and $\mu$ are dominated by L-regular measures and we have $\nu \leq \rho$ on $\mathbf{L}_{2}$ and $\mu \leq\left.\rho\right|_{A\left(L_{1}\right)}$ on $\mathbf{L}_{1}$.

(a) Since $\mathbf{L}_{1}$ is strongly normal $\left.\rho\right|_{A\left(L_{1}\right)} \leq \lambda$ on $\mathbf{L}_{1}$ and then $\left.\rho\right|_{A\left(L_{1}\right)} \in \mathbf{I}_{\sigma}\left(\mathbf{L}_{1}\right)$. Since $\mathbf{L}_{2}$ is $\mathbf{L}_{1}$-countably bounded $\rho \in \mathbf{I}_{\sigma}\left(\mathbf{L}_{2}\right)$, hence $\rho \in \mathbf{I}^{\sigma}{ }_{\mathbf{R}}\left(\mathbf{L}_{2}\right)$.

(b) Since $\mathbf{L}_{1}$ semiseparates $\mathbf{L}_{2}$, we have $\left.\rho\right|_{A\left(L_{1}\right)} \in \mathbf{I}_{\mathbf{R}}\left(\mathbf{L}_{1}\right)$. But since $\mathbf{L}_{1}$ is normal, we must have $\left.\rho\right|_{A\left(L_{1}\right)}=\lambda$, and therefore $\left.\rho\right|_{A\left(L_{1}\right)} \in \mathbf{I}_{\sigma}\left(\mathbf{L}_{1}\right)$.

As in (a), it follows that $\rho \in \mathbf{I}^{\sigma}{ }_{\mathbf{R}}\left(\mathbf{L}_{2}\right)$.

(c) As in (b) we get that $\left.\rho\right|_{A\left(L_{1}\right)} \in \mathbf{I}_{\sigma}\left(\mathbf{L}_{1}\right)$. Since $\mathbf{L}_{2}$ is $\mathbf{L}_{1}$-countably paracompact it follows then that $\rho \in \mathbf{I}_{\sigma}\left(\mathbf{L}_{2}\right)$, hence $\rho \in \mathbf{I}^{\sigma}{ }_{\mathbf{R}}\left(\mathbf{L}_{2}\right)$.

Theorem 6.8. Let $\mathbf{L}_{1} \subset \mathbf{L}_{2}$ be two lattices of subsets of $\mathbf{X}$ and suppose that $\mathbf{L}_{2}$ satisfies condition $(\sigma)$. Then the following are true.

(a) If $\mathbf{L}_{1}$ semiseparates $\mathbf{L}_{2}$ and if $\mathbf{L}_{2}$ is $\mathbf{L}_{1}$-countably bounded, then $\mathbf{L}_{1}$ satisfies condition $(\sigma)$.

(b) If $\mathbf{L}_{1}$ is $\delta$ and $\sigma(\mathbf{L}) \subset s(\mathbf{L})$ and if $\mathbf{L}_{2}$ is $\mathbf{L}_{1}$-countably bounded, then $\mathbf{L}_{1}$ satisfies condition $(\sigma)$.

Proof. Let $\mu \in \mathbf{I}_{\sigma}\left(\mathbf{L}_{1}\right)$ and extend it to $\rho \in \mathbf{I}_{\sigma}\left(\mathbf{L}_{2}\right)$ since $\mathbf{L}_{2}$ is $\mathbf{L}_{1}$-countably bounded.

$\mathbf{L}_{2}$ satisfies condition $(\sigma)$, therefore there exists $v \in \mathbf{I}^{\sigma}{ }_{\mathbf{R}}\left(\mathbf{L}_{2}\right)$ such that $\rho \leq v$ on $\mathbf{L}_{2}$. Consider the restriction $\left.\nu\right|_{A\left(L_{1}\right)}$. If $\mathbf{L}_{1}$ semiseparates $\mathbf{L}_{2}$ (or if $\mathbf{L}_{1}$ is $\delta$ and $\sigma(\mathbf{L}) \subset s(\mathbf{L})$ ), then $\left.v\right|_{A\left(L_{1}\right)} \in \mathbf{I}^{\sigma}{ }_{\mathbf{R}}\left(\mathbf{L}_{1}\right)$ and $\mu \leq\left. v\right|_{A\left(L_{1}\right)}$ on $\mathbf{L}_{1}$.

Therefore $\mathbf{L}_{1}$ satisfies condition $(\sigma)$.

\section{Acknowledgments}

The author is grateful to the referees for exceptional care in reading the original manuscript, for correcting a number of errors, and for improving the presentation of the paper. 


\section{References}

[1] A. D. Alexandroff, Additive set-functions in abstract spaces, Rec. Math. [Mat. Sbornik] N. S. 8 (1940), 307-348.

[2] G. Bachman and P. D. Stratigos, Criteria for $\sigma$-smoothness, $\tau$-smoothness, and tightness of lattice regular measures, with applications, Canad. J. Math. 33 (1981), no. 6, 1498-1525.

[3] __ On general lattice repleteness and completeness, Illinois J. Math. 27 (1983), no. 4, 535561.

[4]_, On measure repleteness and support for lattice regular measures, Int. J. Math. Math. Sci. 10 (1987), no. 4, 707-724.

[5] G. Bachman and A. Sultan, Extensions of regular lattice measures with topological applications, J. Math. Anal. Appl. 57 (1977), no. 3, 539-559.

[6] Representations of linear functionals on spaces of continuous functions, repletions, and general measure extensions, J. Math. Anal. Appl. 67 (1979), no. 2, 277-293.

[7] G. Bachman and M. Szeto, On strongly measure replete lattices and the general Wallman remainder, Fund. Math. 122 (1984), no. 3, 199-217.

[8] G. M. Eid, On normal lattices and Wallman spaces, Int. J. Math. Math. Sci. 13 (1990), no. 1, $31-38$

[9] P. R. Halmos, Measure Theory, Graduate Texts in Mathematics, vol. 18, Springer, New York, 1974.

[10] P.-S. Hsu, Applications of outer measures to separation properties of lattices and regular or $\sigma$ smooth measures, Int. J. Math. Math. Sci. 19 (1996), no. 2, 253-262.

[11] A. M. Koltun, Lattice measures and compactification, Ph.D. Dissertation, Polytechnic University of New York, New York, 1975.

[12] M. E. Munroe, Measure and Integration, 2nd ed., Addison-Wesley, Massachusetts, 1971.

[13] M. Szeto, Measure repleteness and mapping preservations, J. Indian Math. Soc. (N.S.) 43 (1979), no. 1-4, 35-52.

[14] C. Vlad, Lattice separation and properties of Wallman type spaces, Ann. Mat. Pura Appl. (4) 159 (1991), 65-79.

[15] _ On lattice-topological properties of general Wallman spaces, Int. J. Math. Math. Sci. 21 (1998), no. 1, 25-32.

[16] Applications of outer measures to $\sigma$-smoothness and $\tau$-smoothness of measures, J. Math. Sci. (Calcutta) 10 (1999), no. 1, 11-19.

Carmen D. Vlad: Department of Mathematics, Pace University, Pleasantville, NY 10570, USA

E-mail address: cvlad@pace.edu 


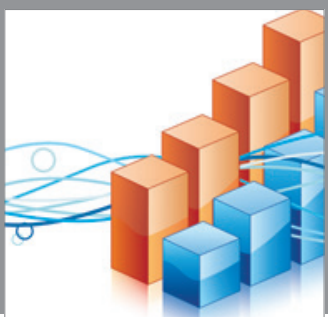

Advances in

Operations Research

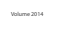

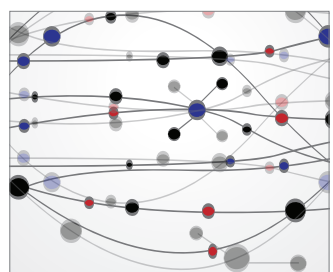

\section{The Scientific} World Journal
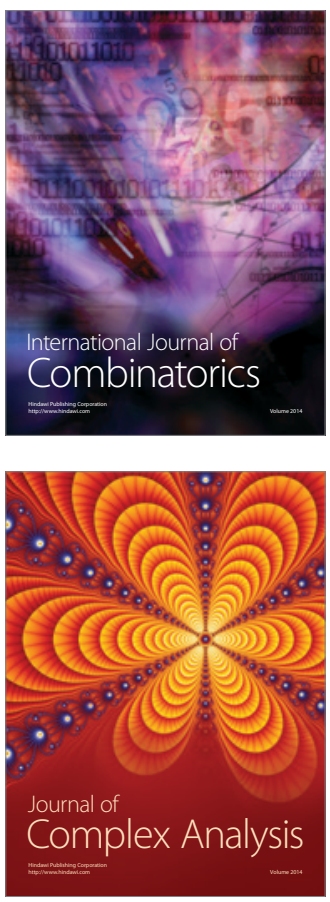

International Journal of

Mathematics and

Mathematical

Sciences
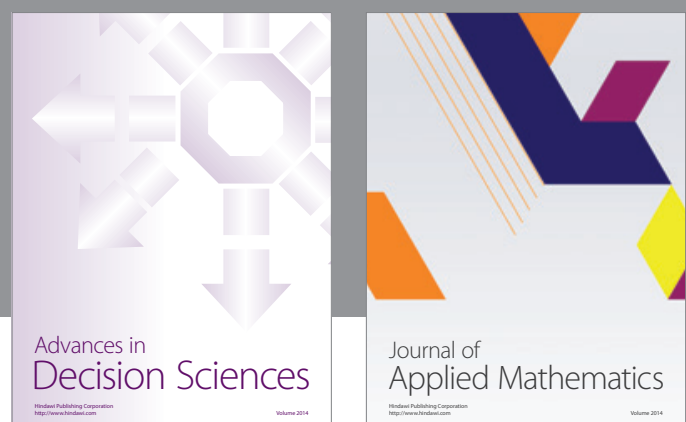

Journal of

Applied Mathematics
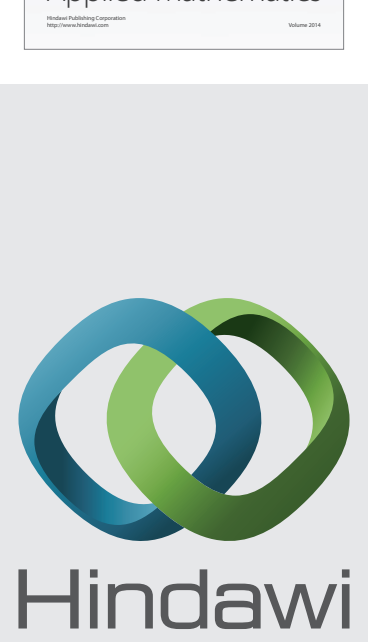

Submit your manuscripts at http://www.hindawi.com
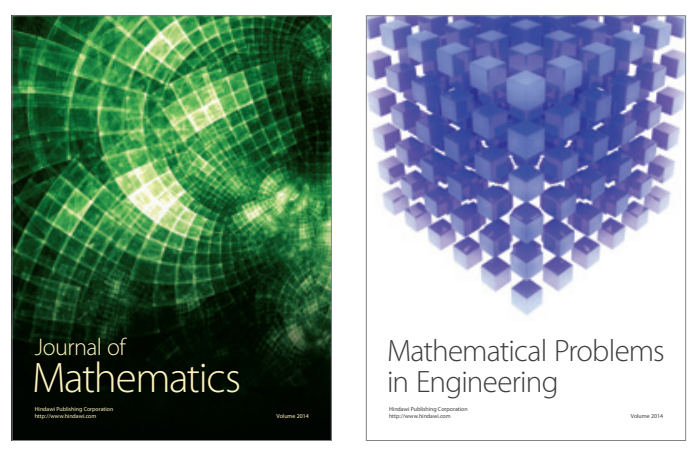

Mathematical Problems in Engineering
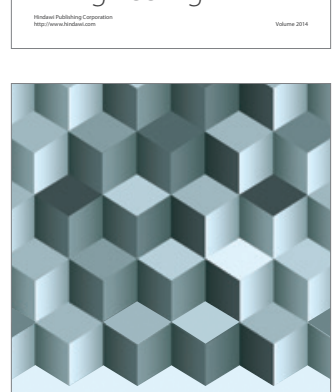

Journal of

Function Spaces
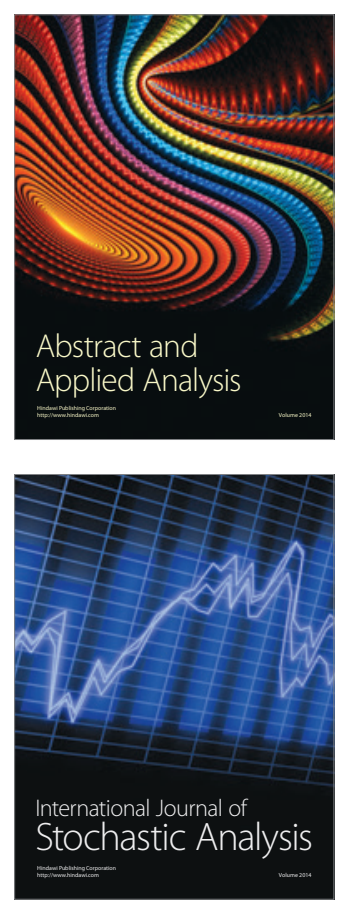

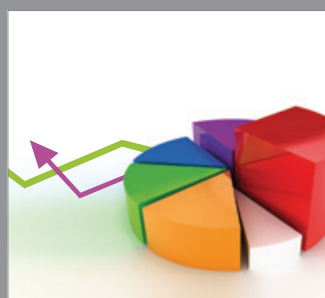

ournal of

Probability and Statistics

Promensencen
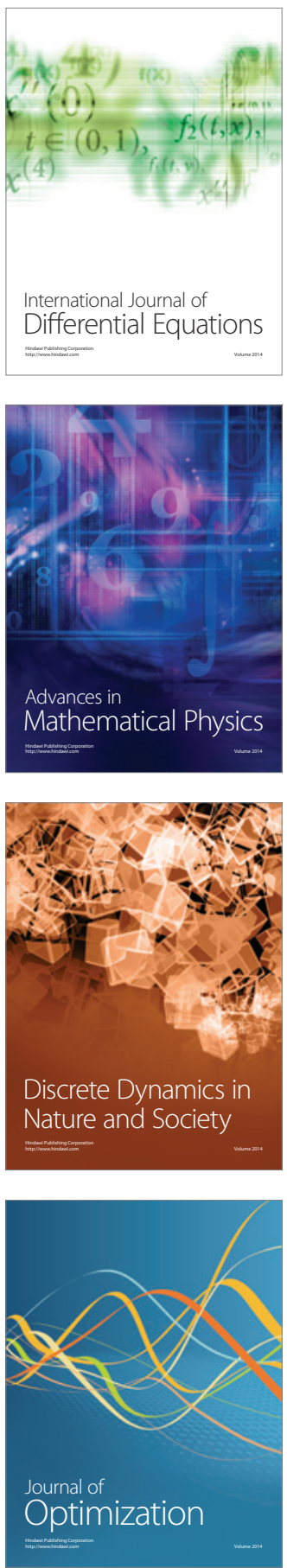